\title{
Stateless Two-Stage Multiple Criteria Scheduling in Nuclear Medicine
}

\author{
Shu-Yen Wan, ${ }^{1}$ Che-Yao Chang, ${ }^{1,2}$ Chun-Ling Wang, ${ }^{1}$ and Kun-Ju Lin ${ }^{3}$ \\ ${ }^{1}$ Department of Information Management, Chang Gung University, Taoyuan 333, Taiwan \\ ${ }^{2}$ Graduate Institute of Business and Administration, Chang Gung University, Taoyuan 333, Taiwan \\ ${ }^{3}$ Department of Nuclear Medicine, Chang Gung Memorial Hospital, Taoyuan 333, Taiwan \\ Correspondence should be addressed to Kun-Ju Lin; lin4857@adm.cgmh.org.tw
}

Received 5 October 2013; Revised 7 January 2014; Accepted 8 January 2014; Published 20 February 2014

Academic Editor: Ching-Ter Chang

Copyright (C) 2014 Shu-Yen Wan et al. This is an open access article distributed under the Creative Commons Attribution License, which permits unrestricted use, distribution, and reproduction in any medium, provided the original work is properly cited.

Examination in nuclear medicine exhibits scheduling difficulties due to its intricate clinical issues, such as varied radiopharmaceuticals for different diseases, machine preparation and length of scan, and patients' and hospital's criteria and/or limitations. Many scheduling methods exist but are limited for nuclear medicine. In this paper, we present stateless two-stage scheduling to cope with multiple criteria decision making. The first stage mostly deals with patients' conditions. The second stage concerns more the clinical condition and its correlations with patients' preference which presents more complicated intertwined configurations. A greedy algorithm is proposed in the second stage to determine the (time slot and patient) pair in linear time. The result shows practical and efficient scheduling for nuclear medicine.

\section{Introduction}

In this paper, a scheduling methodology that considers the patient attributes and clinical preferences is proposed to arrange the patient appointments and examinations in nuclear medicine departments. The objective of the study is to maximize the number of patients to be serviced and thus to maximize resource allocation under intertwined constraints imposed by the patients and the hospital. The proposed stateless two-stage approach alleviates the scheduling complexity by transforming the, by nature, stochastic multiple criteria process into a binary classification problem in the first stage, followed by really making scheduling decisions in the second stage using a linear greedy approach without carrying over the rankings from the first stage. The two-stage decision process coincides with the realistic clinical practices to take into account varied configurations of inpatients and outpatients.

Nuclear medicine is a branch of medical imaging that acquires physiologically functional images by capturing the radiation from nuclides injected in a patient's body and the equipped machines are to collect radiation data and restructure the signals into images that may reflect a person's physiological function $[1,2]$.

Different diseases require varied radioactive nuclides and employ disparate procedures in resource and administration management [3, 4]. Further, machine restriction, startup time, radiopharmaceuticals logistics, and direct care and labor time have complicated the appointment scheduling and have introduced significant difficulty for pure manual processing [3, 5-7]. Imaging procedure management has become a major part of US government expenses and scheduling effectiveness thus depends on articulated management of various criteria and factors [1]. Existing literature on nuclear medicine is limited and most of it emphasizes procedure management $[4,6-8]$.

The upfront issue for appointment scheduling in nuclear medicine is how to arrange most possible patients into limited time slots when machines are available. Patients have various conditions: types of diseases, doctor-ordered next visit time before when prescribed examinations have to be done, available or preferred weekdays, home distance from the hospital, time to request examination arrangement, and so forth. The nuclear medicine departments have their criteria too: 
available time slots, available machines on different days, scan preference in each slot, how many patients can be squeezed in a slot (different slots may have different configurations), and so forth. An effective scheduling is expected to satisfy these intertwined multiple criteria. The multiple criteria in combination thus lead to multiple criteria decision-making problems.

Mohanty and Bhasker proposed a fuzzy decision support method that takes into account multiple attributes to solve Internet business problems [9]. Chang proposed a novel concept of binary behavior in efficiently solving fuzzy programming $[10,11]$. In later efforts, Chang simplified representations of S-shaped membership functions and proposed multiple criteria fuzzy programming with consideration of binary behaviors $[12,13]$.

In clinical applications, Vissers developed a resource allocation scheme to balance the needs from the inpatients and the distributed workload for the nursing staff. Although only few factors were taken into account, the model had its wide applicability [14]. Vlah et al. employed Variable Neighborhood Search strategy to solve inpatient scheduling. Their heuristic approach was proved efficient when the number of patients reaches forty [15]. Chern et al. presented an efficient heuristic algorithm for health examination scheduling in the hospital. The authors examined the waiting time of both examinees and doctors. Detailed configurations are considered while some uncertain conditions were not taken into account. The major difference from our study was that the examination appointments had been done in advance [16]. Vermeulen et al. proposed adaptive resource allocation model to accommodate the dynamic characteristics in hospital scheduling. CT-scan scheduling was specifically investigated in detail. Although not comprehensive, their valuable parameters discussed were also employed and examined in our study $[17,18]$. The other similar work from Vermeulen et al. further investigated the aspects of urgencies and preferences, which are important factors when conducting appointment scheduling [19].

Scheduling in nuclear medicine has its intrinsically more complicated issues. An example is that the radiologists may impose precedence on the scans as per the disinfection requirements. Scans for certain group of patients may have to be scheduled apart from the others. Scheduling for inpatients and that for outpatients are different. Scheduling for both the appointments and examinations exhibits more challenges.

To cope with the complexity of scheduling in nuclear medicine, we propose a two-stage approach. The patients' conditions are considered in the first stage and we employ a slightly remodeled multiple criteria fuzzy programming to choose candidates from the waiting-for-examinationschedule pool. To suit the proposed paradigm for varied clinical cases in nuclear medicine, the two-stage approach is modeled as stateless, meaning that the first-stage ranking will play no roles in the second stage. The hospital or clinical configurations (hence some of the patients' attributes) are considered in the second stage. The second-stage scheduling needs to be more adaptive, adjustable, and dynamic to deal with various criteria/limitation/factors imposed to individual time slots. For each slot, a criterion-patient preference matrix is constructed; all slot-patient preference levels are evaluated and ranked. Finally, we proposed a greedy algorithm to determine patients' allocation in scheduled time slots.

The rest of the paper is structured as follows. In the Methods section, we introduce the proposed methodology. The stateless two-stage approach is detailed. In the Clinical Examples and Results section, we experiment the proposed scheduling method with a downsized realistic clinical example from nuclear medicine, followed by concluding remarks.

\section{Methods}

This work proposes a stateless two-stage scheduling paradigm for nuclear medicine examinations. The first stage takes into account the patients' static and temporal configurations, such as traveling distance to the hospital, severity/emergency of illness, and time left to the scheduled doctor revisit. A multiple criteria fuzzy programming adapted from $[12,13]$ is proposed to determine patients who take precedence to enter the second stage. In the second stage, for a given time slot, the correlated preference/limitation between the scanner and a patient is considered. For example, bone scans take less time and more than one patient can be scheduled into one time slot; patients may prefer specific slots, or, in certain circumstances, lung scans take priority to heart scans. The scheduling is stateless in that the ranking of the first stage is not carried over to the second stage. A two-stage-withmemory scheduling can be deemed as a special case of the proposed paradigm: patients enter the second stage in the ranking order sequentially. An algorithmic greedy approach of the second stage takes linear time.

2.1. First-Stage Scheduling. Chang proposed a novel multiple criteria fuzzy programming to account for binary behaviors in classification associated with various membership functions $[10,12,13]$. A membership function can be considered as a concatenation of a sequence of S-shaped increasing (Figure 1) and decreasing (Figure 2) functions and, therefore, the proposed scheduling solution to the multiple criteria decision problem can be summarized and formulated as follows:

$$
\operatorname{Min} \sum_{i=1}^{M}\left[\sum_{j=1}^{B_{i}} f_{i j}+\alpha_{i}\left(e_{i}^{+}+e_{i}^{-}\right)\right]
$$

such that

$$
\lambda_{i}=\left\{\begin{array}{rl}
\sum_{j=1}^{B_{i}-2}[ & \left.\left(u_{i}\left(b_{i(j+1)}\right)-u_{i}\left(b_{i j}\right)\right) \frac{p_{i j}-p_{i(j+1)}}{b_{i(j+1)}-b_{i j}}\right] \\
& +\left(u_{i}\left(b_{i B_{i}}\right)-u_{i}\left(b_{i\left(B_{i}-1\right)}\right)\right) \\
& \times \frac{p_{i\left(B_{i}-1\right)}}{b_{i\left(B_{i}\right)}-b_{i\left(B_{i}-1\right)}},
\end{array} \quad f_{i j}=p_{i j}\right.
$$




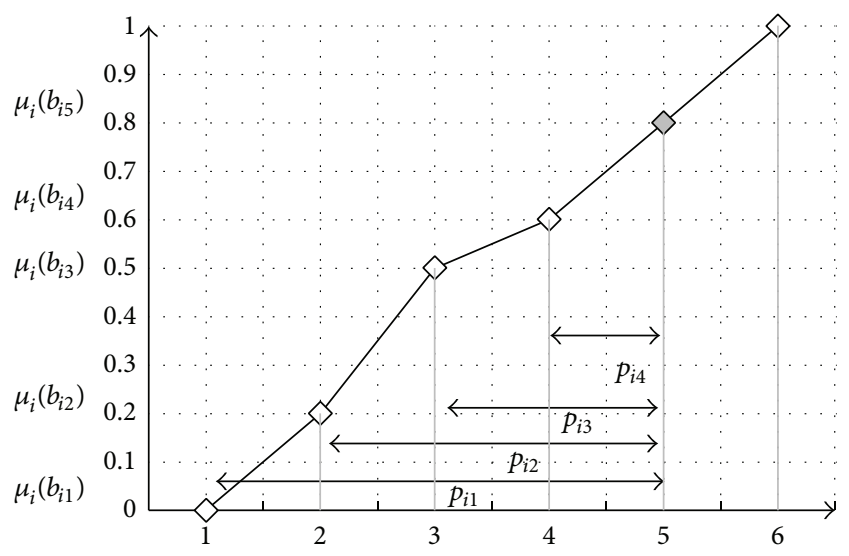

FIGURE 1: Example of open right S-shaped membership function for criterion $i$.

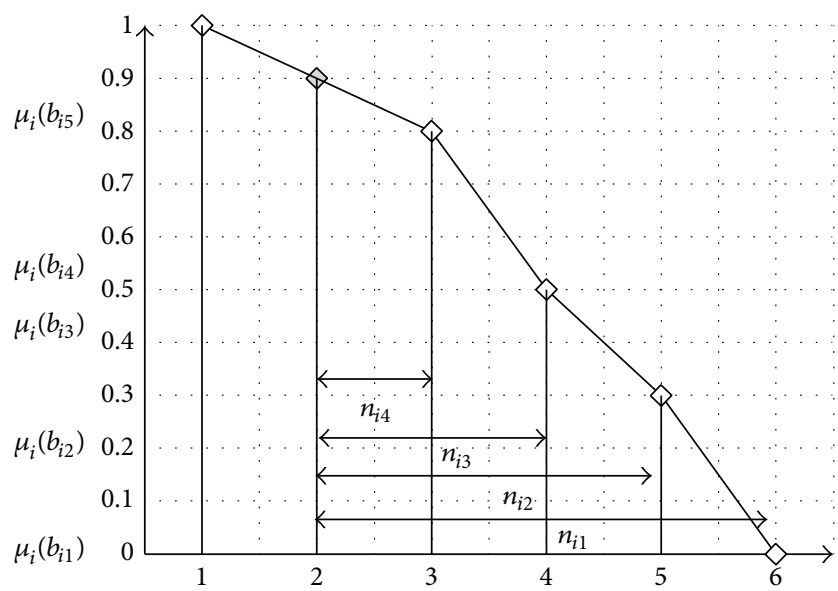

FIgURE 2: Example of open left S-shaped membership function for criterion $i$.

$$
\begin{gathered}
\lambda_{i}-{e_{i}^{+}}^{+}+e_{i}^{-}=1 \\
z_{i}\left(P_{s}\right)-p_{i j}+n_{i j}=b_{i j}, \quad \forall j=1 \cdots B_{i},
\end{gathered}
$$

where $M$ is the number of criteria; $B_{i}$ is the number of break points on the membership function $u_{i}$ for criterion $i ; f_{i j}=\left\{p_{i j}, n_{i j}\right\}$ is the positive or negative deviation, respectively, from the break points $b_{i j} ; e_{i}{ }^{+}$and $e_{i}{ }^{-}$are positive and negative deviations from the highest membership value of $u_{i} ; \lambda_{i}$ is a continuous membership variable used for computation purpose; $\alpha_{i}$ is the weight attached to the sum of the deviations of $\left|\lambda_{i}-1\right| ; z_{i}\left(P_{s}\right)$ is a quantitative measure of criterion/factor/attribute/limitation $i$ with respect to patient $P_{s}, \forall s=1 \cdots S$, for all $S$ patients.

When taking binary behaviors into account, the following constraints must also be satisfied.
For positive deviations,

$$
\begin{aligned}
& 1-\lambda_{i} \leq \sum_{s=1}^{S-1}\left(u_{i}\left(P_{s}\right)-u_{i}\left(P_{s+1}\right)\right) y_{s, i}-m, \\
& y_{s, i} \geq y_{s+1, i}, \quad s=1 \cdots(S-1), \quad y_{s, i}=0 .
\end{aligned}
$$

For negative deviations,

$$
\begin{aligned}
& 1-\lambda_{i} \leq \sum_{s=2}^{S-1}\left(u_{i}\left(P_{s+1}\right)-u_{i}\left(P_{s}\right)\right) y_{s+1, i}-m, \\
& y_{s+1, i} \geq y_{s, i}, \quad s=2 \cdots(S-1), \quad y_{1, i}=0,
\end{aligned}
$$

where $m$ is a small number and $y_{s, i}$ is a binary variable to indicate whether patient $P_{s}$ with respect to criterion $i$ will be selected.

The model could be adjusted by its satisfaction level, as needed, to determine how many patients would be selected as candidates to enter the second stage.

2.2. Second-Stage Scheduling. The goal of the second stage is to assign a patient to its most preferable time slot. Assume that there are $T$ available time slots, $S$ patients, and $R$ criteria. Without loss of generality, the number of patients $(S)$ should be equal to or less than the number of overall patients under consideration for scheduling. For a given time slot $k$, its criterion-patient preference matrix can be represented by

$$
\left[\begin{array}{ccc}
L_{k 11} & \cdots & L_{k 1 S} \\
\vdots & \ddots & \vdots \\
L_{k R 1} & \cdots & L_{k R S}
\end{array}\right]_{R \times S}
$$

where $L_{k i j}$ represent the preference level of the patient $j$ for criterion $i$ with respect to time slot $k$. As the configurations of all criteria may vary significantly, the preference levels are precomputed and stored in a lookup table (LUT) which can dynamically be adapted and extracted according to overall configuration changes. A clinical example is given in the Clinical Example and Result section for further description.

Each patient $j$ for time slot $k$ has, therefore, an evaluated precedence value $E_{k j}$ (or EPV), such that

$$
E_{k j}=\prod_{i=1}^{R} L_{k i j} .
$$

There are totally $(T \times S)$ such evaluated precedence values satisfying $0 \leq E_{k j} \leq 1$. The proposed scheduling performs sorting in decreasing order and removes those whose values are zero; that is, no assignment is possible for certain restricted configurations, to form an EPV list. The final scheduling process implements a greedy approach by searching through the EPV list from the front to the end. Each EPV reveals a specific patient; for example, $E_{35}$ is the evaluated precedence value of patient \#5 for time slot \#3. Whenever a unvisited EPV is visited, if its corresponding patient has not assigned a slot and the requested slot is big enough to accommodate the patient, he/she shall be 
scheduled and be removed from the waiting list. The process continues until no more patients are still waiting in stage two or the EPV list is empty. The patients left behind are required to relax his/her limitation to run the second-stage scheduling again. The proposed algorithm is given in Algorithm 1.

The scheduling process may then restart anew or we may lower the satisfaction level for the first stage, picking up other patients to enter the second stage.

\section{Clinical Example and Results}

To justify the efficacy of the proposed scheduling method, we demonstrate an example adapted from a realistic nuclear medicine case.

Assume that six patients are on the to-be-scheduled list for nuclear medicine examinations. The following factors are considered: days left for doctor revisit, traveling time to the hospital, type of examination, and the patients' availability. Table 1 shows the example configuration.

Preference levels are real numbers between 0 and 1 . When it is closes to the doctor revisit day, the need of scheduled examination is more immediate, as shown in Figure 3. When considering traveling distance to the hospital, the scheduling is usually favorable for those who live afar, as shown in Figure 4 .

By employing the multiple criteria fuzzy programming with binary behavior proposed in [12], we begin with the firststage scheduling and obtain the solution as follows:

$$
\begin{aligned}
& \text { Min } n_{11}+n_{12}+n_{13}+n_{14}+n_{15}+e_{1}^{+}+e_{1}^{-} \\
& +p_{21}+p_{22}+p_{23}+p_{24}+p_{25}+e_{2}^{+}+e_{2}^{-} \\
& \text {s.t. } \quad \lambda_{1}=\left[0.1\left(\frac{n_{11}-n_{12}}{10}\right)+0.2\left(\frac{n_{12}-n_{13}}{6}\right)\right. \\
& +0.3\left(\frac{n_{13}-n_{14}}{4}\right) \\
& \left.+0.1\left(\frac{n_{14}-n_{15}}{3}\right)+0.2\left(\frac{n_{15}}{2}\right)\right] \\
& \lambda_{1}-e_{1}^{+}+e_{1}^{-}=1 \\
& z_{1}(x)-p_{11}+n_{11}=30 \text { for } b_{11} \\
& z_{1}(x)-p_{12}+n_{12}=20 \text { for } b_{12} \\
& z_{1}(x)-p_{13}+n_{13}=14 \text { for } b_{13} \\
& z_{1}(x)-p_{14}+n_{14}=10 \text { for } b_{14} \\
& z_{1}(x)-p_{15}+n_{15}=7 \text { for } b_{15} \\
& \lambda_{2}=\left[0.2\left(\frac{p_{21}-p_{22}}{30}\right)+0.3\left(\frac{p_{22}-p_{23}}{40}\right)\right. \\
& +0.1\left(\frac{p_{23}-p_{24}}{50}\right) \\
& \left.+0.2\left(\frac{p_{24}-p_{25}}{50}\right)+0.2\left(\frac{p_{25}}{50}\right)\right] \\
& \lambda_{2}-e_{1}^{+}+e_{1}^{-}=1 \\
& z_{2}(x)-p_{21}+n_{21}=30 \text { for } b_{21} \\
& z_{2}(x)-p_{22}+n_{22}=60 \text { for } b_{22} \\
& z_{2}(x)-p_{23}+n_{23}=100 \text { for } b_{23} \\
& z_{2}(x)-p_{24}+n_{24}=150 \text { for } b_{24} \\
& z_{2}(x)-p_{25}+n_{25}=200 \text { for } b_{25}
\end{aligned}
$$

$$
\begin{array}{r}
1-\lambda_{1} \leq \sum_{i=1}^{5}\left(u_{\text {time }}\left(P_{i}\right)-u_{\text {time }}\left(P_{i+1}\right)\right) y_{i, 1} \\
-0.00001, \\
y_{i, 1} \geq y_{i+1,1}, \quad i=1,2,3,4, \quad y_{6,1}=0, \\
1-\lambda_{2} \leq \sum_{i=1}^{5}\left(u_{\text {distance }}\left(P_{i+1}\right)-u_{\text {distance }}\left(P_{i}\right)\right) y_{i+1,2} \\
-0.00001, \quad \\
y_{i+1,2} \geq y_{i, 2}, \quad i=2,3,4,5, \quad y_{1,2}=0 .
\end{array}
$$

The problem is solved by using LINGO [20]. How many patients are selected to enter the second-stage scheduling is determined by adjusting the value of $\lambda$ (satisfaction level). When we specify $\lambda_{1}=0.5$ and $\lambda_{2}=0.5$, only one patient $\left(P_{3}\right)$ is chosen. In our case, we set $\lambda_{1}=0.2$ and $\lambda_{2}=0.2$, and four patients $\left(P_{2}, P_{3}, P_{4}\right.$, and $\left.P_{5}\right)$ are chosen to enter the second-stage scheduling. The proposed scheduling process is stateless; therefore, the ranking of $\left(P_{2}, P_{3}, P_{4}\right.$, and $\left.P_{5}\right)$ will not be carried over and the four candidates are equally considered in stage two.

In the second-stage scheduling, we assume there are ten time slots, two slots a day from Monday through Friday Four factors are taken into account: type of examination $\left(f_{1}\right)$, occupancy of the examination $\left(f_{2}\right)$, patient's availability $\left(f_{3}\right)$, and slot popularity factor $\left(f_{4}\right)$.

Type of examination is a clinically frequently referenced factor. A usual scenario is that lung/bone/heart scans are customarily arranged on Monday; the other scans are preferably moved to the other weekdays. In additions lung scans are preferred to heart scans, and heart scans are, in turn, preferred to bone scans. A possible preference lookup table is shown in Table 2.

Occupancy length of examination is another important clinical issue to consider in scheduling. Examination items can take various length of time: 10 minutes at least and some may take up to 40 minutes. To accommodate as many patients as possible, squeezing more than one patient into a single time slot is a usual practice in clinic. Consider a time slot of length 1; if certain examination takes fewer time and may squeeze 5 patients in a slot, each examination has a preference level of 0.2 (or 1/5). If only 2 patients of an examination are permitted in a time slot, each would be assigned a preference level of 0.5 by the proposed scheduling process. A patient's availability is a binary variable (0: unavailable or 1: available). Slot popularity factor tells how competitive the slot is, that is how many patients compete a single slot. A patient gets a preference level 0.25 for a slot if there are four people claiming available.

We thus obtain 40 criterion-patient preference matrices, 10 time slots for 4 patients. The criterion-patient preference matrix for Monday morning (time slot \#1) is:

$$
\begin{aligned}
& P_{2} \\
& f_{1} \\
& f_{2} \\
& f_{3} \\
& f_{4}
\end{aligned} \quad\left[\begin{array}{cccc}
0.8 & 0.8 & 0.5 & 0.2 \\
0 & 0 & 1 & 1 \\
1 & 0 & 1 & 1 \\
0.5 & 0.5 & 0.5 & 0.5
\end{array}\right]
$$




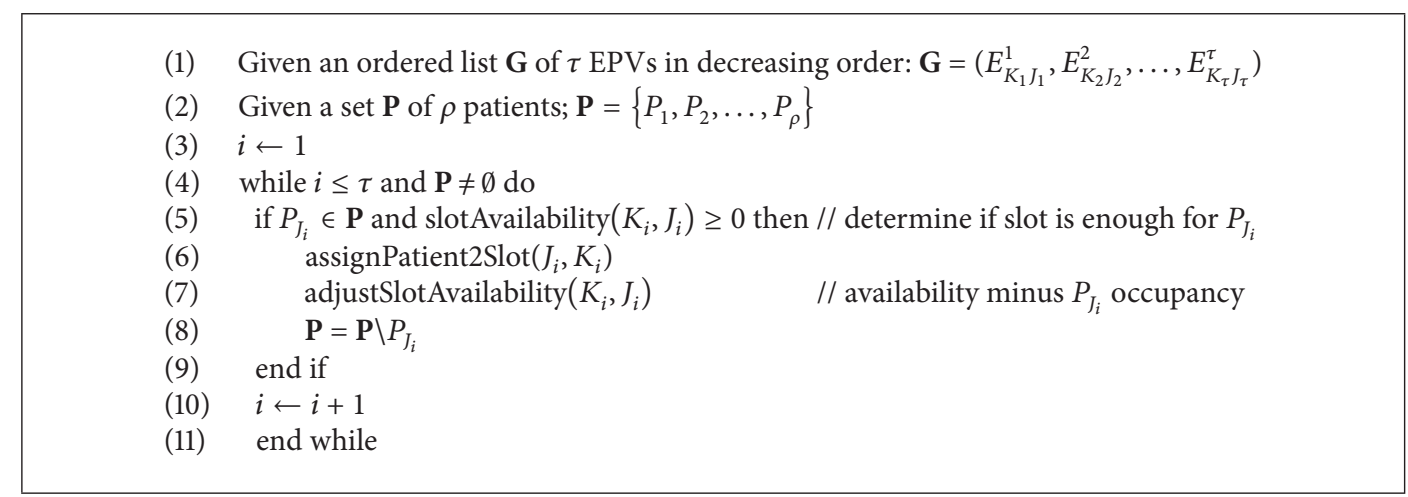

Algorithm 1

TABLE 1: Conditions of the patients and the corresponding preference levels (memberships).

\begin{tabular}{|c|c|c|c|c|c|c|}
\hline Patient & Days to revisit & Traveling distance $(\mathrm{km})$ & Examination & Availability & Preference level (time) & Preference level (distance) \\
\hline$\overline{P_{1}}$ & 5 & 30 & Bone & Mon/Tue, Wed & 1 & 0.1 \\
\hline$P_{2}$ & 7 & 60 & Lung & Mon only & 0.8 & 0.2 \\
\hline$P_{3}$ & 10 & 100 & Lung & Tue/Wed & 0.7 & 0.5 \\
\hline$P_{4}$ & 14 & 150 & Heart & All & 0.4 & 0.6 \\
\hline$P_{5}$ & 20 & 200 & Bone & Mon/Tue & 0.2 & 0.8 \\
\hline$P_{6}$ & 30 & 250 & Heart & Wed/Fri & 0.1 & 1 \\
\hline
\end{tabular}

TABle 2: Precedence example for the radiological examination.

\begin{tabular}{lc}
\hline Type of examination & Preference level in terms of $f_{1}$ \\
\hline Lung & 0.8 \\
Bone & 0.2 \\
Heart & 0.5 \\
The others & 0 \\
\hline
\end{tabular}

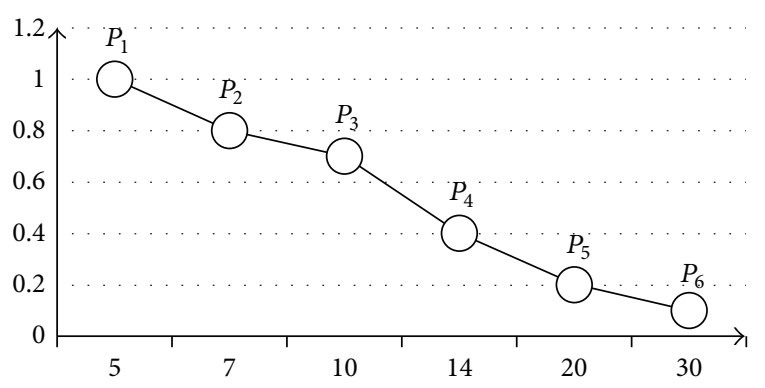

Figure 3: Membership function of the factor: days left to doctor revisit (open left S-shaped decreasing MF).

The evaluated preference values of the four patients for Monday morning are $E_{12}=0, E_{13}=0, E_{14}=0.25$, and $E_{15}=0.1$, respectively. The results show that patient $\# 2$ and patient \#3 will not be assigned in Monday morning slot, while patient \#4 takes priority over patient \#5.

Considering all $40 \mathrm{EPVs}\left(E_{i j} ; \forall i=1 \cdots 10, j=2,3,4,5\right)$, those of value 0 are discarded, while the rest are sorted in descending order and stored in EPV list G. Applying the proposed algorithm, patient \#2 is scheduled for Monday

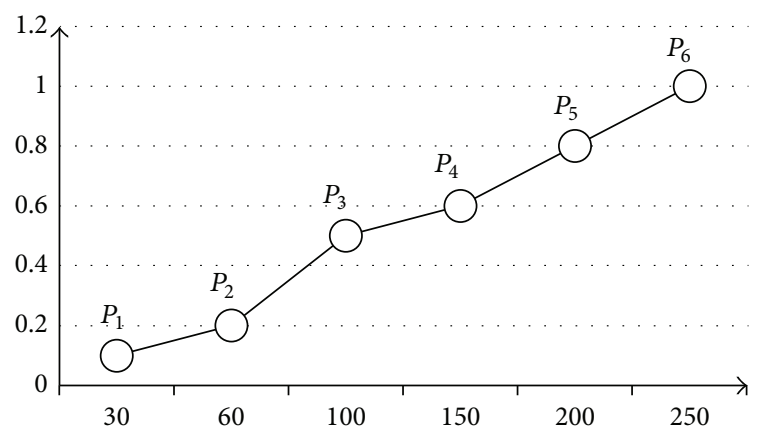

FIGURE 4: Membership function of the factor: home distance to the hospital (open right S-shaped increasing MF).

afternoon, Patient \#3 Tuesday morning, Patient \#4 Monday morning, Patient \#5 Tuesday afternoon.

\section{Discussion and Conclusions}

We have proposed an adaptive scheduling for nuclear medicine examination. The two-stage paradigm alleviates the complexity of a variety of criteria raised by the patients, hospital, administration office, staff, and others. Further, the stateless approach offers more general and flexible applications, as "stated" approach can be deemed as a special case by adjusting the value of $\lambda$ as needed. The methodology of the first stage is mainly adapted from the work of Chang which yields stable, efficient, and sensible ranking results with help of the LINGO software. The computation of the second stage 
is linear. The proposed algorithm to determine slot-patient allocation is efficient, thanks to its greedy approach.

In our example, four patients all get scheduled. We have also implement the proposed scheduling strategy on larger number of inpatients and outpatients to meet their appointment and examination needs and showed efficacy. The proposed algorithm does not have intrinsic limitations. However, if a patient asks for improbable requirements under a difficult circumstance (e.g., specifically asking for certain weekday when it was already assigned to the other higher-priority patient) or the number of patients is more than the possible slots/machines to provide services, the patient is required to relax its criteria by taking back to restart the second stage or even rolled back to the first stage for full reconsideration. It meets a common clinical practice in the department of nuclear medicine. Otherwise, after the first stage has achieved it optimization in ranking and classification, the nature of the proposed second-stage greedy algorithm is able to arrange all possible appointments, without actually inducing any limitations.

Radiological scheduling in the department of nuclear medicine exhibits its stochastic and dynamic characteristics. Outpatients may arrive or call at any time to raise the need for appointments. Inpatients may also submit their requests in a bulk amount. The proposed two-stage approach can help and solidify the current clinical practice by first identifying the most urgent and priority cases in batch (in the first stage) and then requesting for sequential arrangements (in the second stage). Later arrivals may join the queued patients and receive responsive attention.

\section{Conflict of Interests}

The authors declare that there is no conflict of interests regarding the publication of this paper.

\section{Acknowledgments}

This work was partially supported by Chang Gung Memorial Hospital, Taiwan, under Grant CRRPD5C0251 and by National Science Council, Taiwan, under Grant NSC101-2218E-182A-001.

\section{References}

[1] D. Delbeke and G. M. Segall, "Status of and trends in nuclear medicine in the United States," The Journal of Nuclear Medicine, vol. 52, supplement 2, pp. 24S-28S, 2011.

[2] E. Pérez, L. Ntaimo, C. O. Malavé, C. Bailey, and P. McCormack, "Stochastic online appointment scheduling of multistep sequential procedures in nuclear medicine," Health Care Management Science, vol. 16, no. 4, pp. 281-299, 2013.

[3] M. W. Carter and S. D. Lapierre, "Scheduling emergency room physicians," Health Care Management Science, vol. 4, no. 4, pp. 347-360, 2001.

[4] M. A. Centeno, R. Giachetti, R. Linn, and A. M. Ismail, "A simulation-ILP based tool for scheduling ER staff," in Proceedings of the Winter Simulation Conference (WSC '03), vol. 2, pp. 1930-1938, New Orleans, La, USA, December 2003.
[5] D. Gupta and B. Denton, "Appointment scheduling in health care: challenges and opportunities," IIE Transactions, vol. 40, no. 9, pp. 800-819, 2008.

[6] D. Sinreich and O. Jabali, "Staggered work shifts: a way to downsize and restructure an emergency department workforce yet maintain current operational performance," Health Care Management Science, vol. 10, no. 3, pp. 293-308, 2007.

[7] S. Suthummanon, V. K. Omachonu, and M. Akcin, "Applying activity-based costing to the nuclear medicine unit," Health Services Management Research, vol. 18, no. 3, pp. 141-150, 2005.

[8] S. A. Erdogan and B. Denton, "Dynamic appointment scheduling of a stochastic server with uncertain demand," INFORMS Journal on Computing, vol. 25, no. 1, pp. 116-132, 2013.

[9] B. K. Mohanty and B. Bhasker, "Product classification in the Internet business-a fuzzy approach," Decision Support Systems, vol. 38, no. 4, pp. 611-619, 2005.

[10] C.-T. Chang, "Binary behavior of fuzzy programming with piecewise linear membership functions," IEEE Transactions on Fuzzy Systems, vol. 15, no. 4, pp. 710-717, 2007.

[11] C.-T. Chang, "Multi-choice goal programming," Omega, vol. 35, no. 4, pp. 389-396, 2007.

[12] C. T. Chang, "On product classification with various membership functions and binary behaviour," Journal of the Operational Research Society, vol. 65, no. 1, pp. 141-150, 2013.

[13] C.-T. Chang, "An approximation approach for representing Sshaped membership functions," IEEE Transactions on Fuzzy Systems, vol. 18, no. 2, pp. 412-424, 2010.

[14] J. M. H. Vissers, "Patient flow-based allocation of inpatient resources: a case study," European Journal of Operational Research, vol. 105, no. 2, pp. 356-370, 1998.

[15] S. Vlah, Z. Lukač, and J. Pacheco, "Use of VNS heuristics for scheduling of patients in hospital," Journal of the Operational Research Society, vol. 62, no. 7, pp. 1227-1238, 2011.

[16] C.-C. Chern, P.-S. Chien, and S.-Y. Chen, "A heuristic algorithm for the hospital health examination scheduling problem," European Journal of Operational Research, vol. 186, no. 3, pp. 11371157, 2008.

[17] I. B. Vermeulen, S. M. Bohte, S. G. Elkhuizen, J. S. Lameris, P. J. M. Bakker, and J. A. la Poutré, "Adaptive optimization of hospital resource calendars," in Artificial Intelligence in Medicine, vol. 4594 of Lecture Notes in Computer Science, pp. 305-315, 2007.

[18] I. B. Vermeulen, S. M. Bohte, S. G. Elkhuizen, H. Lameris, P. J. M. Bakker, and H. la Poutré, "Adaptive resource allocation for efficient patient scheduling," Artificial Intelligence in Medicine, vol. 46, no. 1, pp. 67-80, 2009.

[19] I. B. Vermeulen, S. M. Bohte, P. A. N. Bosman, S. G. Elkhuizen, P. J. M. Bakker, and J. A. la Poutré, "Optimization of online patient scheduling with urgencies and preferences," in Artificial Intelligence in Medicine, vol. 5651 of Lecture Notes in Computer Science, pp. 71-80, 2009.

[20] L. Schrage, LINGO Release 9.0, LINDO Systems, Chicago, Ill, USA, 2004. 


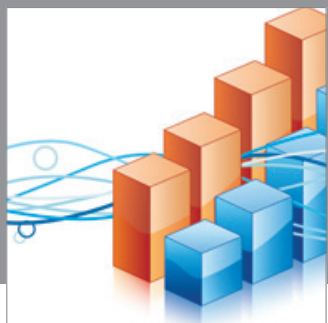

Advances in

Operations Research

mansans

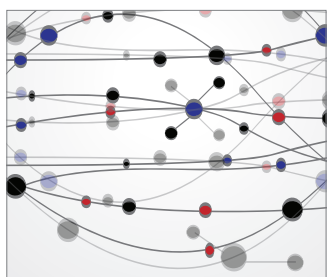

The Scientific World Journal
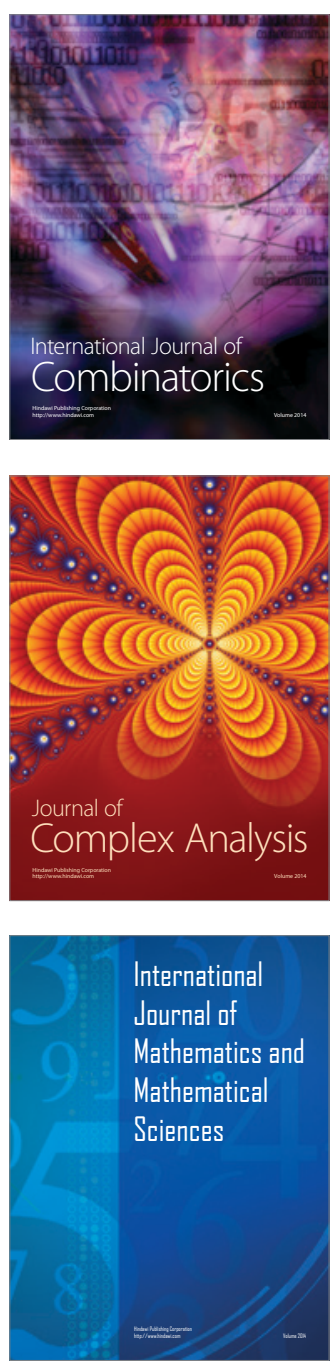
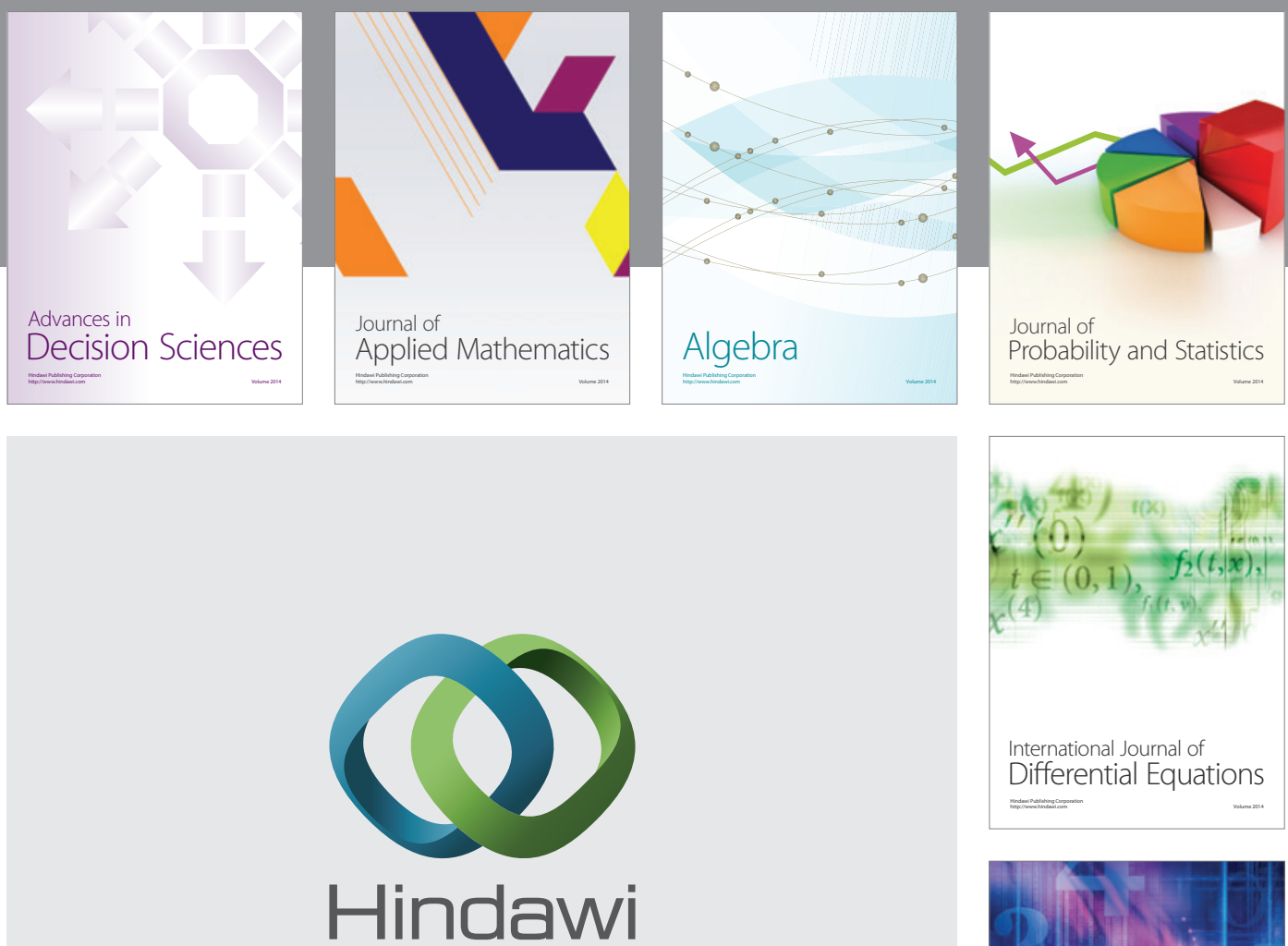

Submit your manuscripts at http://www.hindawi.com
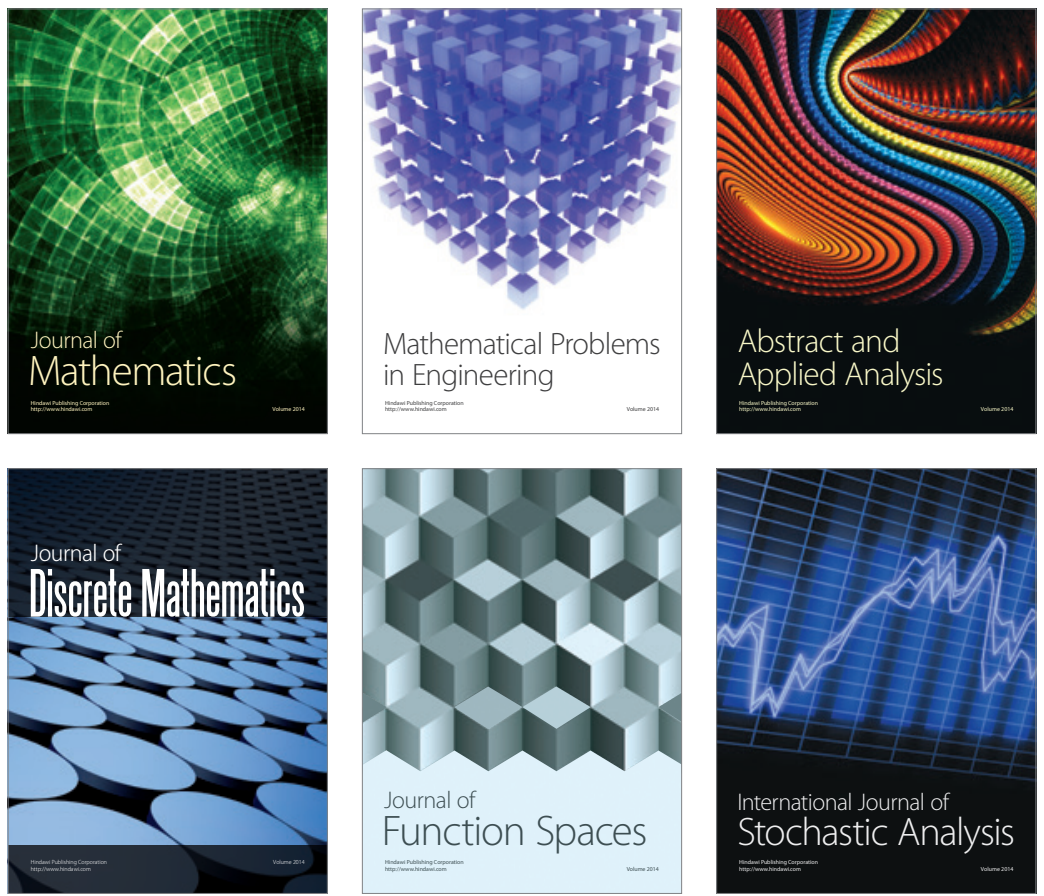

Journal of

Function Spaces

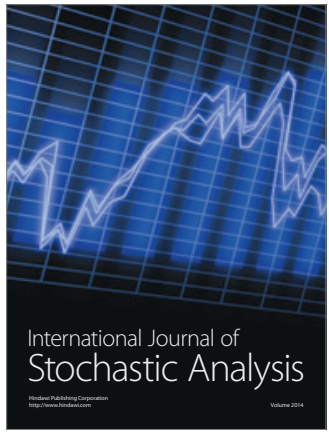

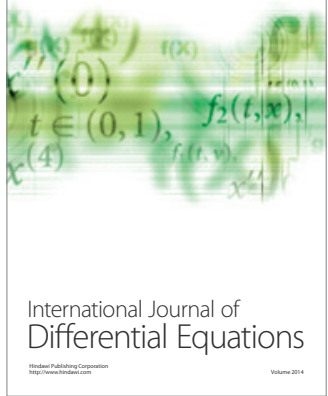
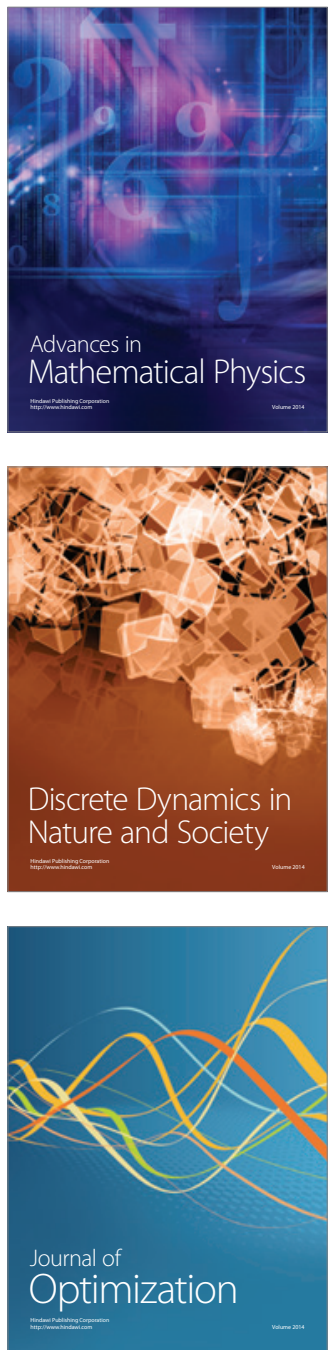